\title{
Uncertainty and Option Value in Land Allocation Problems
}

VINCENZINA MESSINA and VALENTINA BOSETTI

Bosetti@disco.unimib.it

DISCo - Dipartimento di Informatica Sistemistica e Comunicazione, Università degli Studi di Milano

Bicocca, Edificio U7, Via Bicocca degli Arcimboldi 8, 20126 Milano, Italy

\begin{abstract}
In this paper we are concerned with modelling techniques for evaluating development and conservation opportunities when dealing with investment decisions involving environmental resources management. In this context, highly characterized by both environmental and economic uncertainty, we emphasize the importance of capturing the flexibility of different investment strategies. In particular, we discuss a discrete model that includes both environmental and economic uncertainty treated using an integrated approach. Decision analysis techniques and option pricing theory are jointly applied to evaluate development versus conservation opportunities. In our analysis, we take into account how uncertainty interacts with two types of irreversibility: sunk costs associated with investment in developing decisions, including environmental and social costs, as well as sunk costs associated with environmental regulation and conservation. The Quasi Option Value, QOV, is used to derive decision rules that account for different levels of flexibility of land allocation possibilities.
\end{abstract}

Keywords: uncertainty, option value, environment, land allocation

\section{Introduction}

In the presence of uncertain future events, the possibility to postpone development investment decisions often leads to alternative strategies than those which use traditional discounted cash flows approaches. Indeed, the availability of new information may partially resolve uncertainties over time, thus making it profitable to wait and act in the light of it. This flexibility becomes even more valuable when we are faced with irreversible decisions. In order to take into account the level of flexibility of different investment strategies, we will use the concept of the Quasi Option Value $(Q O V)$, that is the extra value that can be captured by performing a fully dynamic analysis of the decision problem.

The issue of irreversibility, uncertainty and environmental policy has been largely discussed in the last three decades. From the first definition of the $Q O V$ given by Arrow and Fisher (1974), this key concept has been extended by several authors, among others, Conrad (1980), Hanemann (1989), Krutilla and Fisher (1975) and Coggins and Ramezani (1998). In particular, Conrad showed that the $Q O V$ is equivalent to the value of information, Hanemann emphasized the conditionality upon no development in the first period, Coggins and Ramezani dealt with the issue of the discount rate to be used and applied the "risk neutral" probability method in the valuation of option value. 
In this paper we extend the discrete model developed in Coggins and Ramezani (1998) to include a set of decision problems that are particularly relevant in the European context, where decision makers are more concerned with land recovery expenditures and conservation efficiency rather than with wilderness preservation. Indeed, in our optimal land allocation model, we consider, in addition to the status quo possibility, two further investment opportunities: the development alternative and the land recovery/conservation alternative (Natural Park). Hence, we allow for two different sources of irreversibility: sunk costs associated with investment in developing decisions, including environmental and social costs due to the permanent degradation of resources, as well as sunk costs associated with environmental regulation and conservation investment plans.

These irreversible choices have to be made in the presence of two relevant types of uncertainty: economic and environmental. Indeed, uncertainty can either be related to future social and financial costs/benefits or to the evolution of the environmental scenario. Thus, it is useful to introduce both these two sources of randomness in the model. In the development case, we consider uncertainty concerning future demand for the good produced, that is market uncertainty. While, in the conservation case, the decision maker is uncertain about the environmental quality level that the Natural Park will turn out to be. We call this environmental quality uncertainty and we assume it depends on a vector of environmental parameters that are not known until after the investment is undertaken. In fact, until investment in on-site research and in land recovery is undertaken, the parameters which make up the "ecological value index" are partially or completely unknown and one can only deal with subjective probabilities, calculated by experts considering the information that is available.

Market uncertainties are dealt with using the option pricing approach (Dixit and Pindyck, 1994; Trigeorgis, 1996; Cortazar, Schwartz, and Salinas, 1998), that values real investment projects attempting to replicate their return and risk characteristics through a portfolio of existing assets. If we imply no arbitrage opportunities, the resulting risk neutral probabilities can be applied to calculate the expected present value of the investment. Environmental uncertainties are a source of project specific risk and, therefore, cannot be entirely diversified. Hence, they are dealt with by computing certainty equivalents, applying subjective probabilities and the decision maker's utility function, as suggested in Smith and McCardle (1998, 1999) and Smith and Nau (1995). This approach leads to an integrated methodology which combines decision analysis techniques with option pricing theory. We represent the problem as a two-period optimal stopping problem (Dixit and Pindyck, 1994), where stopping corresponds to investing, while continuation corresponds to waiting for information to resolve part of the uncertainty. The rest of the paper is organised as follow. In section 2 we introduce the decision process framework for the discussed problem by considering market uncertainty as well as environmental uncertainty. Section 3 discusses the inclusion of environmental uncertainty valuation in the overall procedure. Section 4 is devoted to a sensitivity analysis of critical initial values and numerical analysis. Our conclusions are presented in section 5. 


\section{Decision process framework}

\subsection{Market uncertainty}

In this paper we extend the typical decision framework, in which the main issue is to assess whether and when it is optimal to develop (extract) a natural land area (a natural resource), to the case when a decision maker has to deal with a partially degraded area that can be re-converted to a "natural" state. This is often the case in European countries. In order to choose the best strategy the decision maker has to deal with a threefold problem. She can either decide to leave the land as it is, Status $Q u o(S Q)$ or to develop the area through an initial investment, in order to undertake some profitable project, Development $(D V)$. In addition, she can also decide to reconvert it to a completely preserved state. We will call this third investment opportunity Natural Park (NP), even though it has a more general meaning. Choosing the $S Q$ opportunity, the only source of profit is the amenity value accruing every year from recreational use. We will assume this value to be constant, however, the model could be easily extended to consider the case when the amenity value decreases over time according to a decay rate. This may often be the case when no regulation or enforcement of the area have been undertaken. The $S Q$ state is characterized by complete flexibility, thus the model also evaluates the option to invest either in the $D V$ or in the $N P$ projects in the future. Moreover, this state does not imply commitment to sunk costs (e.g., research, re-conversion of habitat degradation, active protection). On the contrary, the other two states involve an initial investment and annual maintenance costs. The Development alternative would result in a stream of random revenues throughout the operating life of the project. This investment decision implies the irreversible sacrifice of any amenity value as well as of any other potential value sources related to the conserved site. In this case the NP option is ruled out. Finally, deciding to preserve the land as a Natural Park would produce a stream of constant revenues due to the ecological services arising from the completely preserved land in addition to the stream of revenues accruing from the amenity value. In this section we will assume this value to be constant and known with certainty. In section 2.2 we will allow for more realistic cases dealing with the uncertainty concerning the $N P$ yearly revenue. Let $r$ denote the constant risk-free interest rate and $R$ be the compounding factor, $R=(1+r)$.

In the $S Q$ state, the present value of the land over $n$ years is:

$$
\bar{A}=\sum_{i=0}^{n} \frac{A}{R^{i}}
$$

where $A$ is a constant amenity value accruing from the $S Q$ state at each year $t$. When computing the present value for an indefinite time horizon, i.e., $i \in[0, \infty)$ equation (1) becomes:

$$
\bar{A}=\sum_{i=0}^{\infty} \frac{A}{R^{i}}=A \frac{R}{(R-1)} .
$$




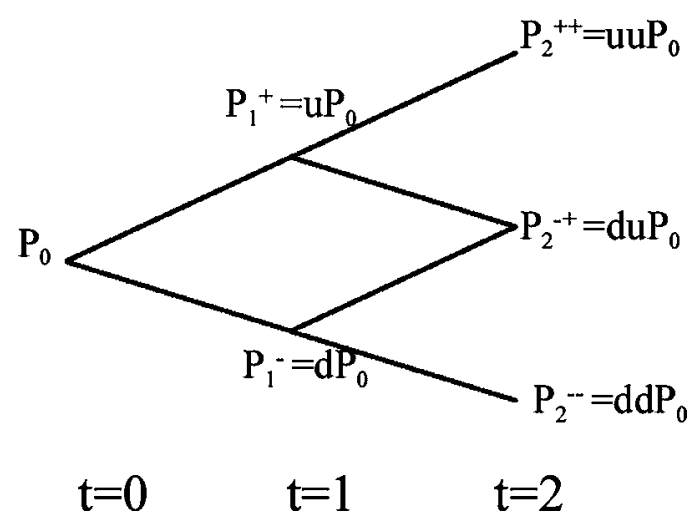

Figure 1. Binomial tree.

In the Development state the initial investment for instantaneous construction of the project is $I_{D}>0$. The present value of the stream of annual constant operating cost, $c$, is given by

$$
C=\sum_{i=0}^{n-1} \frac{c}{R^{i}}=c \frac{R^{n}-1}{R^{n-1}(R-1)},
$$

where $n$ coincides with the operating life of the project.

Assuming for the sake of simplicity that the project will yield one unit of good per year, then the yearly revenue will be given by the output price of the produced good that is assumed to follow a stationary multiplicative random walk. Denoting the output price at time $t$ as $P_{t}$, then at time $t+1$ it will either rise to $P_{t+1}^{+}=u P_{t}$ (where $u>1$ ) or decrease to $P_{t+1}^{-}=d P_{t}$ (where $0<d<1$ ), with probability $q$ and $1-q$, respectively, with $q \in(0,1)$. Moreover, we require $d<R<u$ to rule out profitable risk-less arbitrage opportunities.

Therefore, the price follows a process that can be described by a binomial tree with parameters $\left(P_{0}, u, d, q\right)$, see figure 1 .

Thus, given $P_{0}$, the expected value of $P_{t}$ at time $t>0$, will be given by

$$
E\left[P_{t}\right]=(q u+(1-q) d)^{t} P_{0},
$$

where $E$ is the expectation operator with respect to probability $q$.

We denote the present value of the expected stream of revenues accruing from the $n$ operative years of the $D V$ project, calculated in the first period $(t=0)$, as $P_{0}^{D}$, and this is given by

$$
P V_{0}^{D}=\sum_{i=0}^{n-1} \frac{E\left[P_{i}\right]}{R^{i}}
$$

Denoting

$$
\mu=q u+(1-q) d
$$




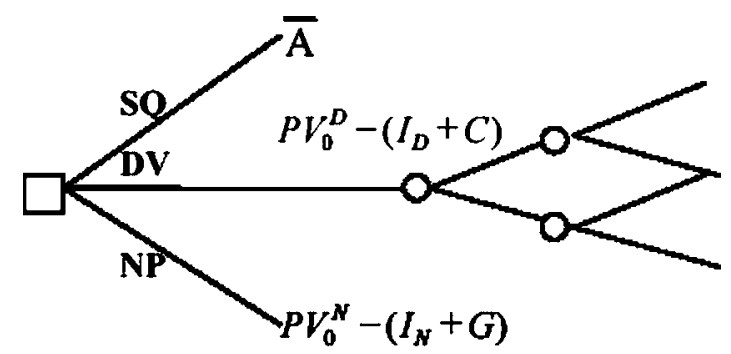

$\mathfrak{t}=\mathbf{0}$

Figure 2. Inflexible modelling decision tree.

we obtain

$$
P V_{0}^{D}\left(P_{0}, q\right)=P_{0}\left[\frac{R^{n}-\mu^{n}}{R^{n-1}(R-\mu)}\right] .
$$

The third alternative, the Natural Park, is characterized by an initial investment, $I_{N}$, and a stream of annual constant operating cost, $g$. The present value, computed for an indefinite time horizon is given by

$$
G=\sum_{i=0}^{\infty} \frac{g}{R^{i}}=g \frac{R}{(R-1)} .
$$

We firstly consider the revenues accruing from the $N P$ option as a stream of constant yearly cash flow, $N=A+E Q$, where $E Q$ is a positive constant value, representing the increased environmental quality characteristics of the site. Then, the present value of the $N P$ can be once again calculated as

$$
P V_{0}^{N}(N)=\sum_{i=0}^{\infty} \frac{N}{R^{i}}=N \frac{R}{(R-1)}
$$

Let us first analyze the problem applying the traditional discounted cash flow rule, without considering the flexibility of postponing decisions.

According to the traditional Expected Net Present Value (ENPV) decision rule (see the decision tree in figure 2), the value of the land area is given by the following statement:

$$
W_{E N P V}=\max \left[\bar{A}, P V_{0}^{D}\left(P_{0}, q\right)-\left(I_{D}+C\right), P V_{0}^{N}(N)-\left(I_{N}+G\right)\right] .
$$

Now, let us consider the possibility to postpone any irreversible decision. The decision tree in figure 3 represents this more flexible decision process, which takes into account future information regarding the price $P_{t}$ and embodies it in the evaluation.

In the $S Q$ state the decision maker is free to switch to one of the two investments in a second period. Let us, as an example, suppose the land has been left in the $S Q$ state 


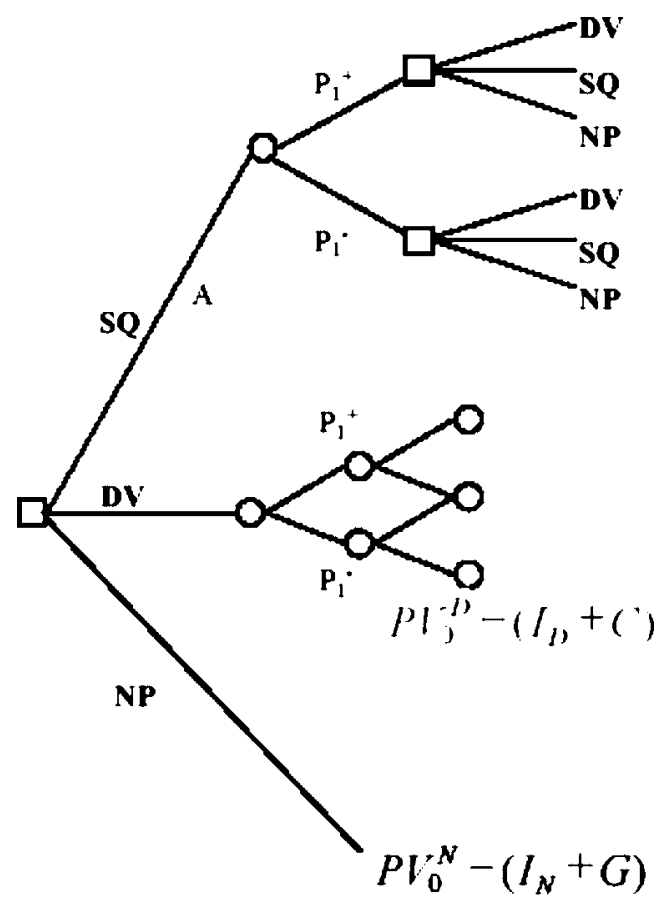

$t=0 \quad t=1$

Figure 3. Flexible modelling decision tree.

for the first period. The only revenue will be the $S Q$ amenity value, $A$. While, in the second period, the availability of new information concerning the price $P_{1}$ will leave the decision maker the possibility to choose her strategy according to the maximization criterion, whichever state of nature has occurred. We can now calculate the value of land in period $t=0$ as

$$
W_{0}^{S}=A+\frac{q W_{1}^{+}+(1-q) W_{1}^{-}}{R},
$$

where the second term of the right-hand side of equation (11) is the expectation value, with respect to the probability $q$ of the quantities

$$
W_{1}^{+}=\max \left[W_{2}^{S}, P V_{1}^{D}\left(P_{1}^{+}, q\right)-\left(I_{D}+C\right), P V_{1}^{N}(N)-\left(I_{N}+G\right)\right]
$$

and

$$
W_{1}^{-}=\max \left[W_{2}^{S}, P V_{1}^{D}\left(P_{1}^{-}, q\right)-\left(I_{D}+C\right), P V_{1}^{N}(N)-\left(I_{N}+G\right)\right] .
$$


Consequently, the decision whether to develop in the first period or delay any investment is based on the value that gives $W_{0}^{*}$, where

$$
W_{0}^{*}=\max \left[P V_{0}^{D}-\left(I_{D}+C\right), P V_{0}^{N}-\left(I_{N}+G\right), W_{0}^{S}\right] .
$$

Since, from definitions (2) and (11) it follows that $W_{0}^{S} \geqslant \bar{A}$, then $W_{0}^{*}$ can also be expressed

$$
W_{0}^{*}=\max \left[W_{E N P V}, W_{0}^{S}\right] .
$$

Indeed, when $W_{E N P V}$ is greater than $W_{0}^{S}$ it means that one of the two investment decisions should take place immediately following the rule given in (10). Whereas, when $W_{0}^{S}$ is greater, waiting is the optimal solution and no sunk costs should be undertaken.

Note that (15) is the Bellman equation for this problem, see (Dixit and Pindyck, 1994), and can be more generally stated as

$$
\begin{aligned}
& W_{t}^{*}=\max \left[W_{E N P V}, W_{t}^{S}\right], \\
& W_{0}^{*}=\max \left[W_{E N P V}, A+R^{-1} E\left[W_{t+1}^{*}\right]\right] .
\end{aligned}
$$

This problem presents itself as an optimal stopping problem (Dixit and Pindyck, 1994; Oksendal and Yaozhong, 1998), where stopping corresponds to invest either in the $D V$ or in the NP project, while continuation corresponds to do not commit to any sunk cost.

We will assume that the decision maker's investment decision can be delayed up to time period, say, $T$ such that afterwards the two investment options will expire. This may often be the case if we consider that political mandates are timed by expiring dates and that investment opportunities are often exclusive for limited periods. In other words, we may think $S Q$ as a portfolio formed by a risk free perpetual bond plus an American option on two different assets (namely, projects $N P$ and $D V$ ) with expiry date $T$. Beyond time period $T$, if the option is not exercised, the only value will be the amenity value, $\bar{A}$. Thus at time period $T$,

$$
W_{T}^{S}=\bar{A} .
$$

It is now possible to calculate the value of the option and to consider the optimal exercise decision by working backwards from date $T$, applying at each step the principle of optimality stated in (16). For some initial critical value of $P_{0}$ it will be optimal to immediately stop and rule out the option, i.e., for $P_{0} \geqslant P_{0}^{*}$ it will always be optimal to invest at $t=0$, while for $P_{0}$ such that $P_{0}^{\prime} \leqslant P_{0}<P_{0}^{*}$ continuation will be the optimal strategy. (We will see that for values below $P_{0}^{\prime}$ the optimal strategy is to invest immediately in $N P$.) Similarly, we can define critical values for $N_{0}$. The $S Q$ choice is optimal provided that neither $P_{0}$ or $N_{0}$ reach the barriers $P_{0}^{*}$ and $N_{0}^{*}$, respectively. In the present work, the $Q O V$ corresponds to the difference, when positive, between the traditionally measured value of the land and the value calculated by using the dynamic model, such that

$$
Q O V=\max \left[0, W_{0}^{*}-W_{E N P V}\right]
$$


The $Q O V$ can capture the value regarding waiting for the availability of new information before making any decision.

\subsection{Environmental quality uncertainty}

Up to this point we have made the assumption that the $N P$ revenues are a constant stream of cash flow, known from period zero. A more realistic approach is to assume that it is not possible to know the environmental quality of a site and the efficiency of the recovery process beforehand. In particular, the environmental quality of an area will depend on a series of uncertain attributes of the site, such as biodiversity, rareness, size of the area, naturalness, level of representation of the inhabitant species (i.e., their uniqueness, etc.). These attributes may be combined in an overall evaluation index of the site. In this work we follow a criteria-based evaluation method based on (Gaston et al., 1998) and, in particular, we consider here three possible states of nature, ${ }^{1}$ each characterized by a prior probability measure.

(1) When either size is insufficient or the unnaturalness of the site is classified as permanent, due to previous damage, the $N P$ value, say, $N_{L}$ is low (with probability $p_{L}$, $\left.0 \leqslant p_{L} \leqslant 1\right)$.

(2) When the site attributes are combined to give an high value, the investment is efficient and the $N P$ value, say, $N_{H}$ is high (with probability $p_{H}, 0 \leqslant p_{H} \leqslant 1-p_{L}$ ).

(3) When the site attributes are combined to give a middle value, the $N P$ value, say, $N_{M}$ is a medium range, such that $N_{M}, N_{L}<N_{M}<N_{H}$ with probability $p_{M}=$ $\left.1-p_{L}-p_{H}\right)$.

Prior probabilities of each environmental quality index state are given by experts or deduced from similar studies. In figure 4, we add this new source of uncertainty, environmental quality uncertainty, represented by black nodes to distinguish it by market uncertainties affecting the $D V$ price process, represented by white nodes. In the next section we discuss how this new source of uncertainty can be included in the overall valuation procedure.

\section{Valuation methodology}

We still have not made any specific assumption neither on the probability distribution nor on the discount rate to be used in the valuation. It is not sensible, though, to discount uncertain cash flows using the risk-less discount rate, $r$, without making any specification.

However, to value the $D V$ investment, we can assume that there exists a complete market for the produced good and, therefore, the associated risk can be perfectly hedged by trading securities. Investment can take place at time period $t=0,1, \ldots, T$ and market uncertainty is resolved at periods $t=0,1, \ldots, T, \ldots, \infty$, thus waiting gives us revised information about the initial price of the project. In a more rigorous way we 


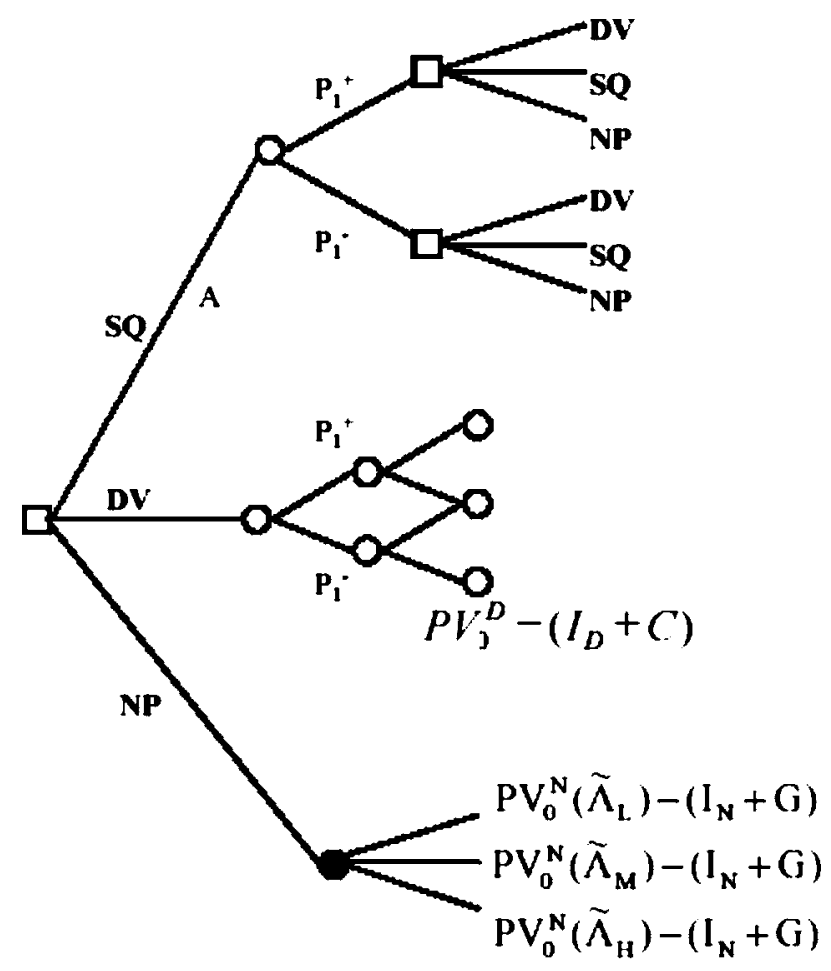

$\mathbf{t}=\mathbf{0} \quad \mathbf{t}=\mathbf{1}$

Figure 4. Decision tree with specified uncertainty types.

can say that the decision maker's state of information at each period $t$ is denoted by $\Im_{t}$, formally modelled as elements of a filtration on a suitably defined probability space.

As most of the real options literature assumes, the decision maker can either decide to invest in the project (all-or-nothing decision) or to invest in shares of securities at market prices, as well as lending and borrowing money at the risk-free interest rate $r$.

Four assumptions on the securities market should be met. Firstly, there are no arbitrage opportunities. Secondly, the market is complete, i.e., market uncertainties can be hedged by trading securities. Thirdly, the market is efficient and does not depend on private information and, finally, there are no transaction costs. Under these assumptions, we can always construct a portfolio whose payoffs exactly replicate the payoffs of the $D V$ project (Eichberger and Harper, 1997). Thus, we can determine the unique "riskneutral" distribution such that the current market price of a vector of securities, $s(0)$, that generates a random dividend stream, $c(t)$, is given by the expression

$$
s(0)=\sum_{t=0}^{T} \frac{E_{\pi}[c(t)]}{(1+r)^{t}},
$$


where $E_{\pi}$ is the expectation with respect to the "risk-neutral" probability, $\pi$ (with $\pi \in(0,1)$ ), and $c(t)$ equals the random stream of revenues arising from the project. Whenever the riskiness of a project is diversifiable, it is possible to compute the value of the project applying the "risk-neutral" probability distribution and using the risk-free interest rate. By using (20) to compute the "risk-neutral" probability distribution for the $D V$ and the $N P$ alternatives, we obtain: ${ }^{2}$

$$
\hat{q}=\frac{(1-d)}{(u-d)} .
$$

It is easy to check that, for such values of $\hat{q}$, the value of $\mu$ as defined in (6), is equal to one. Therefore, the risk-neutral expected value of the $D V$ alternative, given in (7) can now be expressed as

$$
P V_{0}^{D}\left(P_{0}\right)=P V_{0}^{D}\left(P_{0}, \hat{q}\right)=P_{0}\left[\frac{R^{n}-1}{R^{n-1}(R-1)}\right] .
$$

This result entails that, when a project is invested in, then the decision maker has the opportunity to contract with a buyer the selling price of the entire stream of the produced good, fixing it at the investment-time price. The second source of uncertainty, i.e., environmental quality uncertainty, will not be resolved until the $N P$ investment is undertaken. This type of uncertainty cannot be hedged by tradable securities. Hence, in order to include it in the model, a traditionally used approach is the capital-asset-pricing model that produces a risk-adjusted discount factor, according to the risk category of the project. However, this is not the best procedure when the level of risk of a project is not comparable with that of other known projects and when dealing with long time horizon projects. An alternative approach to deal with project specific uncertainty has been developed by Smith and Nau (1995) and applied to evaluate investments in the field of oil and gas management, see (Smith and McCardle, 1998, 1999). The procedure involves the use of subjective prior probabilities, regarding possible future states, which are combined with the decision maker's intertemporal consumption preferences. The value of a project is considered as the buying price, that is the amount that the decision maker would be willing to pay for participation in the project. The buying price is computed as the lump-sum equating the maximum expected utility that would be achieved with participation in the project and the one that would be achieved without participation, see (Smith and Nau, 1995) for a more theoretical discussion. The buying price can be computed easily if the utility function describing the decision maker intertemporal preferences is assumed to be continuous, strictly increasing and strictly concave. Moreover, for the constistency and separation theorems to hold, the utility function should satisfy the property of additivity independence ${ }^{3}$ and the $\Delta$-property. ${ }^{4}$ Without loss of generality and for the sake of concreteness we can assume that the utility function takes the form:

$$
U\left(x_{t}\right)=-\exp \left(-\frac{x_{t}}{K_{t}}\right),
$$


where $K_{t}$ is the decision maker's effective risk tolerance defined as

$$
K_{t}=\sum_{i=t}^{T} \frac{\rho_{i}}{R^{i-t}} .
$$

Hence, $K_{t}$ is the sum of the discounted future decision maker's $i$ th's period risk tolerance, $\rho_{i}$.

Given expert probabilities and the decision maker's utility function, we can compute the effective certainty equivalent, $E C E_{i}$, associated with the $N P$ investment alternative by using the expression

$$
E C E_{i}\left[N P V_{t}^{N}\left(N_{y}\right)\right]=-K_{t} \ln E\left[\exp \left(-\frac{\left[N P V_{t}^{N}\left(N_{y}\right)\right]}{K_{t}}\right)\right],
$$

where $y=L, M, H$ and $N P V_{t}^{N}\left(N_{L}\right), N P V_{t}^{N}\left(N_{M}\right), N P V_{t}^{N}\left(N_{H}\right)$ represent the net present value of the $N P$ project in the three possible scenarios of environmental quality, respectively, $E$ is the expected value with respect to the subjective probability and $K_{t}$ is the decision maker's effective risk tolerance. Now we can integrate both valuation procedures. This involves the generation of the prices tree and the backwards valuation, starting at the end of the tree, by applying a standard dynamic programming "rollback" procedure. In particular, at each node representing market uncertainty, the present value will be calculated by using the risk-neutral probabilities, $E_{\hat{q}}$, and the risk-free interest rate; at each node representing environmental quality uncertainty, the effective certainty equivalent, $E C E_{i}$, will be calculated by using the subjective probability and then subtracting the initial investment; finally, at each of the decision nodes the best strategy will be chosen according to the maximization criterium. If assumptions on market completeness and on preferences hold, this integrated procedure gives the buying price of the land. Firstly, let us consider the value of the land according to the traditional ENPV rule, say, $\widehat{W}_{E N P V}$ such that it can be expressed

$$
\widehat{W}_{E N P V}=\max \left[\bar{A}, P V_{0}^{D}\left(P_{0}\right)-\left(I_{D}+C\right), E C E_{0}\left[N P V_{t}^{N}\left(N_{y}\right)\right]-I_{N}\right],
$$

where $y=L, M, H$. Secondly, the flexibility in the valuation procedure can be expressed considering the value of the $S Q$ state at time $t$, say, $\widehat{W}_{t}^{S}$

$$
\widehat{W}_{t}^{S}=A+\frac{\hat{q} \widehat{W}_{t+1}^{+}+(1-\hat{q}) \widehat{W}_{t+1}^{-}}{R},
$$

where:

$$
\widehat{W}_{t}^{+}=\max \left\lfloor\widehat{W}_{t+1}^{S}, \widehat{P V}_{t+1}^{D}\left(P_{t+1}^{+}\right)-\left(I_{D}+C\right), E C E_{t}\left[N P V_{t}^{N}\left(N_{y}\right)\right]-I_{N}\right\rfloor,
$$

and

$$
\widehat{W}_{t}^{-}=\max \left\lfloor\widehat{W}_{t+1}^{S}, \widehat{P V}_{t+1}^{D}\left(P_{t+1}^{-}\right)-\left(I_{D}+C\right), E C E_{t}\left[N P V_{t}^{N}\left(N_{y}\right)\right]-I_{N}\right\rfloor
$$

where $y=L, M, H$. Note that the expressions (27) and (28) denote the value of the option for both price scenarios at $t=1$. Similarly to the expressions shown in the 
previous section, the value of land, at $t=0$ can be computed recursively by applying the principle of optimality, such that, given the terminal condition $\widehat{W}_{T}^{S}=\bar{A}$, it results

$$
\widehat{W}_{0}^{*}=\max \left[\widehat{W}_{E N P V}, \widehat{W}_{0}^{S}\right] .
$$

Again, the $Q O V$ captures the opportunity of avoiding either the irreversible development choice or the flexible commitment to the $N P$ sunk costs. Its value can be expressed

$$
Q O V=\max \left[0, \widehat{W}_{0}^{*}-\widehat{W}_{E N P V}\right] .
$$

\section{Sensitivity analysis and a numerical example}

In this section we give a numerical example in order to analyze how the two different strategies, computed by applying the traditional and the integrated approaches, vary with the initial value of the two investments, $P_{0}$ and $N_{0}$, respectively. In particular, we aim to characterize the trigger values, for which the two approaches coincide. These cutoff values divide the continuation from the stopping region. Moreover, to account for the uncertainty that may affect different parameters in the model, we analyze how these trigger values are influenced by changes in each of the parameters. Let us suppose that the parameters in the model are defined as given in the table 1 and that decisions can be undertaken at $t=0,1,2$.

In example 1 we consider how strategies change, varying the initial value of the $D V$ investment, $P_{0}$, while keeping constant the value of the $N P$ choice, $N_{0}$. Similarly, in example 2, we let $N_{0}$ change while we keep $P_{0}$ constant.

Example 1. We consider the trends of $\widehat{W}_{E N P V}, \widehat{W}_{0}^{*}$, and $Q O V$, as a function of $P_{0}$, assuming that all the other parameters are fixed. In particular, figure 5 refers to the case of $N_{0}=300$. Until the initial $D V$ price reaches the value $P_{0}^{\prime}$, the traditional rule coincides with our integrated flexible approach and the suggested strategy is to immediately invest in the $N P$ option. The kink in the $\widehat{W}_{0}^{*}$ curve, in point $P_{0}^{\prime}$, captures the value of future period development opportunities for high market states, therefore the "wait and see" strategy should now be an optimal one. In point $P_{0}^{\prime \prime}$ the $\widehat{W}_{E N P V}$ curve starts to increase because the traditional rule indicates that the optimal strategy is now immediate development, while, according to the flexible approach, the strategy "wait and see" is still the optimal one. The cut-off point, $P_{0}^{*}$ represents the threshold value separating the continuation (waiting) region from the stopping (investing in $D V$ ) region. The $Q O V$ represents the maximum amount a rationale decision maker would accept to pay for the right to delay any decision. This value becomes zero above the cut-off value.

Table 1

\begin{tabular}{lccccccccccccccc}
\hline Parameter & $r$ & $n$ & $u$ & $d$ & $\hat{q}$ & $I_{D V}$ & $c$ & $\mu$ & $I_{N P}$ & $p_{H}$ & $p_{M}$ & $N_{H}$ & $N_{L}$ & $g$ & $K_{0}$ \\
Value & 0.1 & 10 & 1.3 & 0.8 & 0.4 & 1000 & 50 & 1 & 800 & 0.4 & 0.5 & 1.4 & 0.9 & 35 & 200 \\
\hline
\end{tabular}




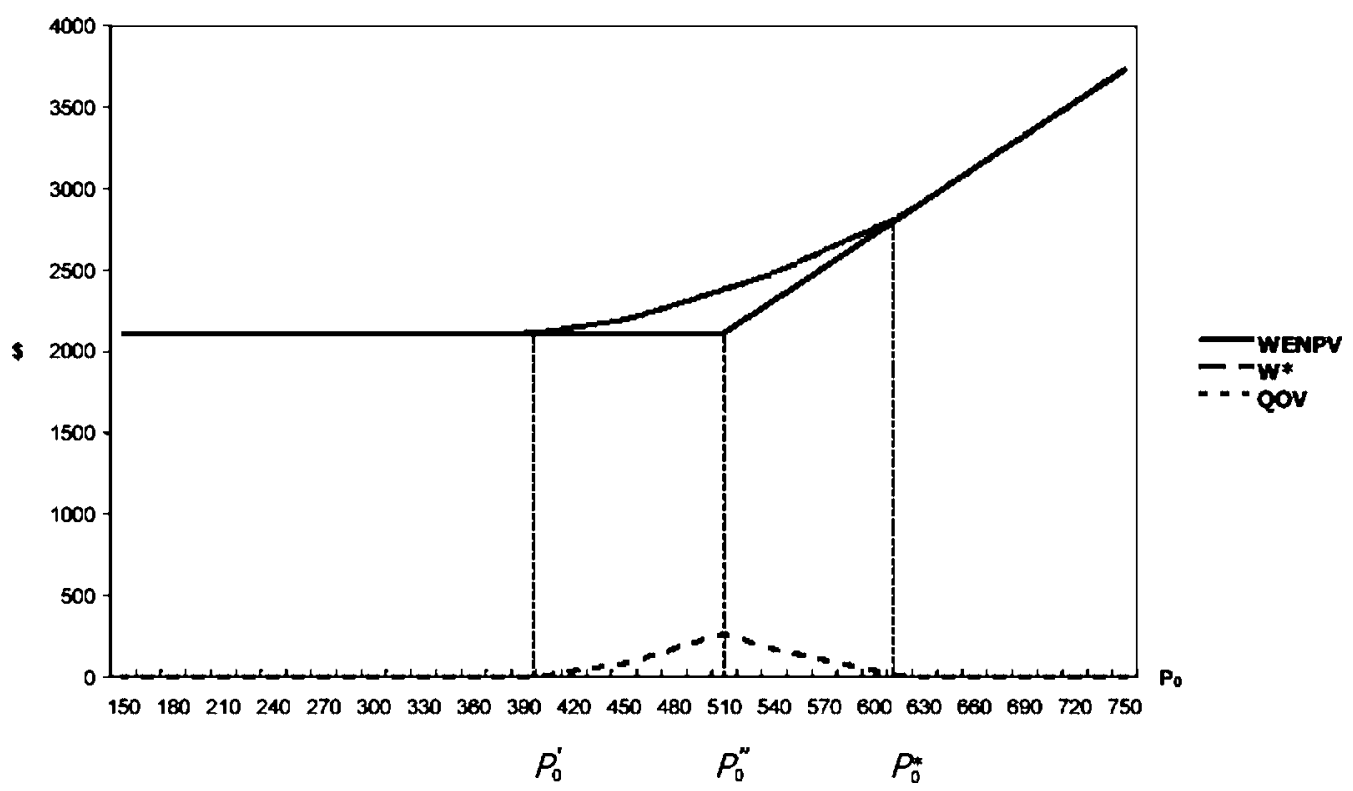

Figure 5. Varying $P_{0}$ for $N_{0}=300$. The dashed line represents $\widehat{W}_{0}^{*}$, the thick line, $\widehat{W}_{E N P V}$ and the dotted line $Q O V$.

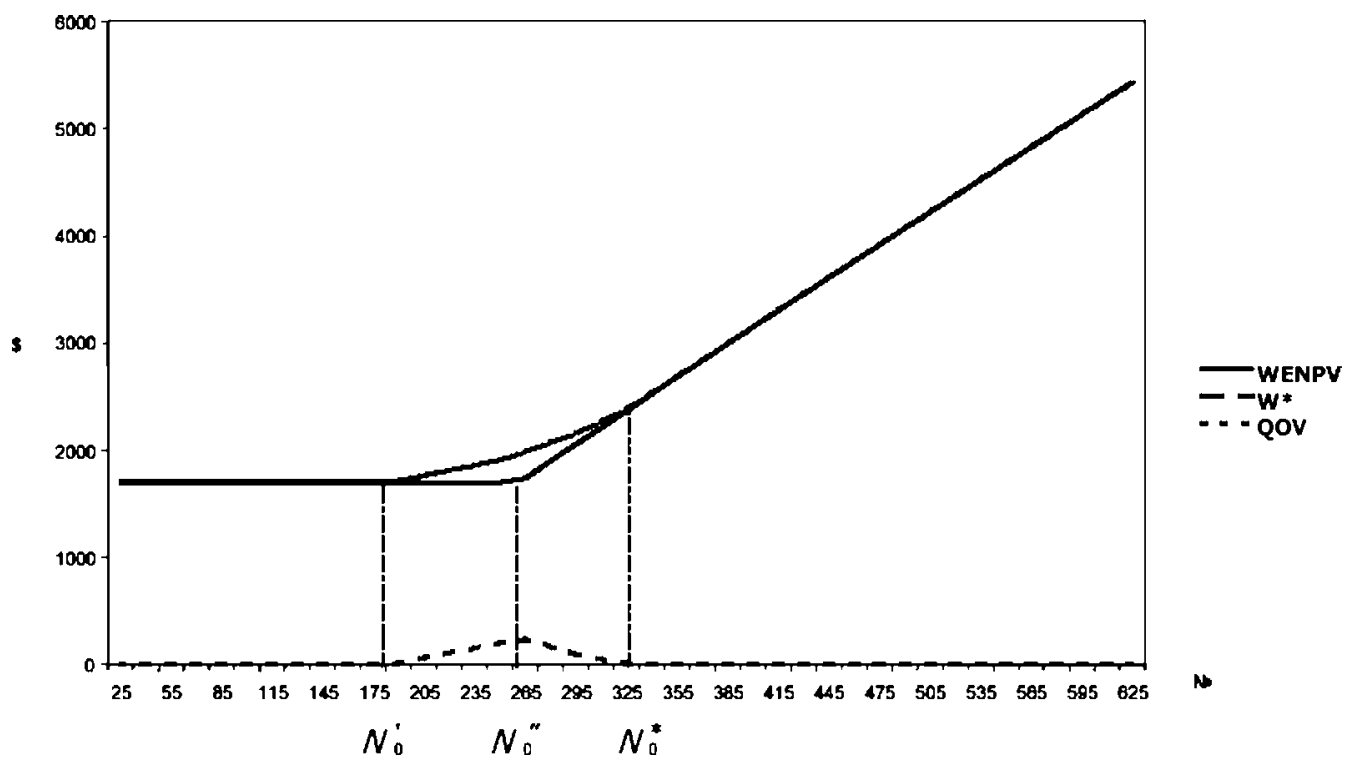

Figure 6. Varying $P_{0}$ for $N_{0}=450$. The dashed line represents $\widehat{W}_{0}^{*}$, the thick line, $\widehat{W}_{E N P V}$ and the dotted line $Q O V$.

Example 2. Again we leave all the parameters fixed and consider the trends of $\widehat{W}_{E N P V}$, $\widehat{W}_{0}^{*}$, and $Q O V$, as a function of $N_{0}$ assuming $P_{0}=450$. As it is depicted in figure 6 , 

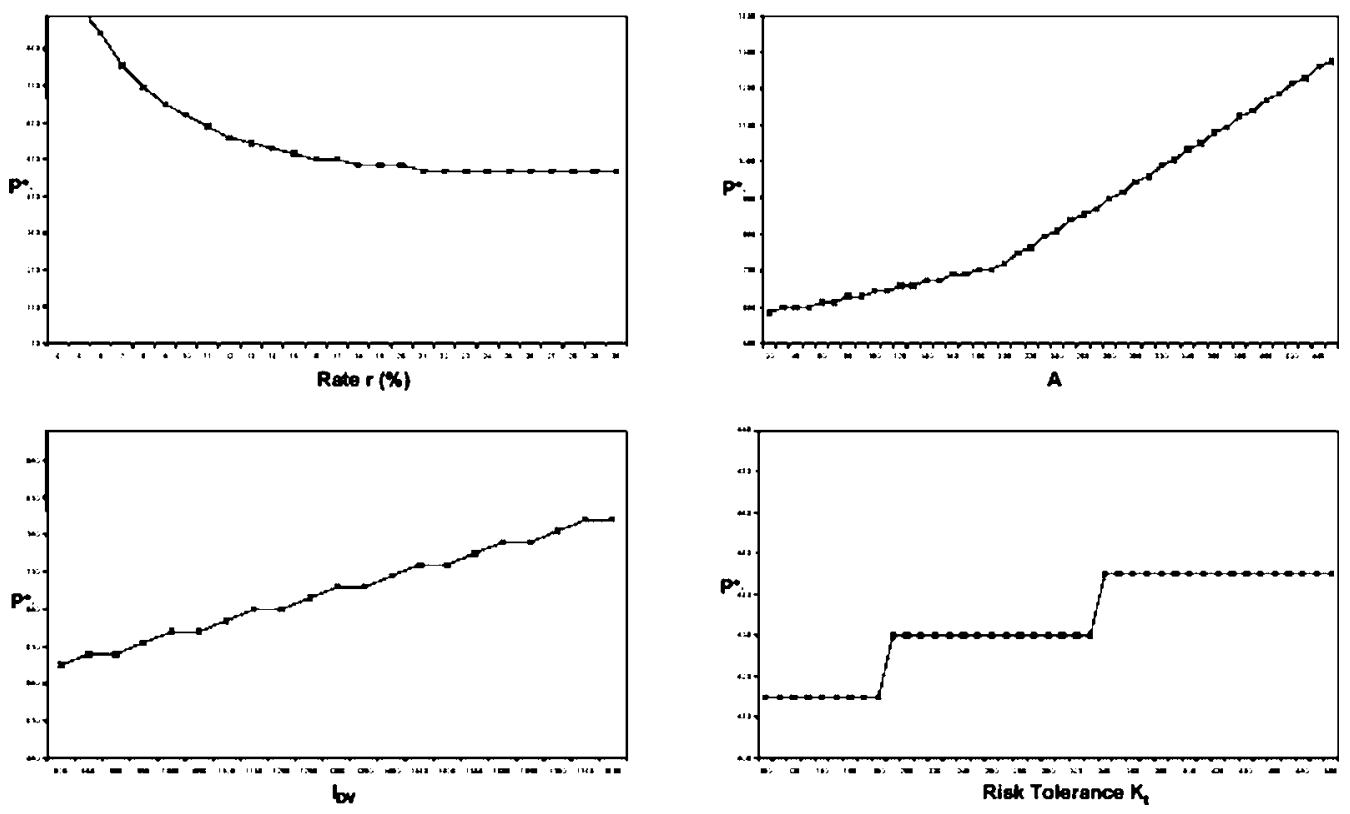

Figure 7. Sensitivity analysis for $P_{0}^{*}$.

the best strategy for very low values of $N_{0}$ is to develop immediately, according to both approaches. In $N_{0}^{\prime}$, the $\widehat{W}_{0}^{*}$ value begins to capture the possibility of investing in the $N P$ alternative, future states of nature being favourable, therefore the strategy "wait and see" should now not be the optimal one. As a consequence, the $\widehat{W}_{0}^{*}$ curve starts to lie above the $\widehat{W}_{E N P V}$ one, thus, generating a positive $Q O V$. From the value $N_{0}^{\prime \prime}$ on, the ENPV rule would suggest to commit to the $N P$ investment, while, according to our approach, it is just at $N_{0}=N_{0}^{*}$ that the $N P$ investment becomes the optimal strategy. Therefore, up to this cut-off point, $N_{0}^{*}$, the $Q O V$ captures the value of not committing to sunk costs and just wait to the second period to take any decision.

We now analyze how these trigger values $P_{0}^{*}$ (see figure 7) and $N_{0}^{*}$ (see figure 8), separating the continuation from the stopping region, may vary with some of the model parameters, all remaining parameters being fixed. In particular, we consider how these values change in respect to: $A$, the first year value of the $S Q$ alternative, $I_{D}$, the $D V$ investment, $I_{N}$, the $N P$ investment, the risk free discount rate, $r$, and the effective risk tolerance factor, $K_{t}$.

$P_{0}^{*}$ is positively correlated with $A$ and with $I_{D}$. In other words, the barrier that the initial price has to reach in order to have immediate development gets higher as the $S Q$ alternative becomes more profitable and as the $D V$ initial investment increases. $P_{0}^{*}$ decreases with $r \in(0.01,0.2)$. In fact, the $D V$ project produces revenues just in the next $n$ years, thus it is less affected by an increase in the discount rate. Finally, an increase in the risk tolerance factor positively affects the $N P$ present value, thus increasing the $D V$ trigger value. 

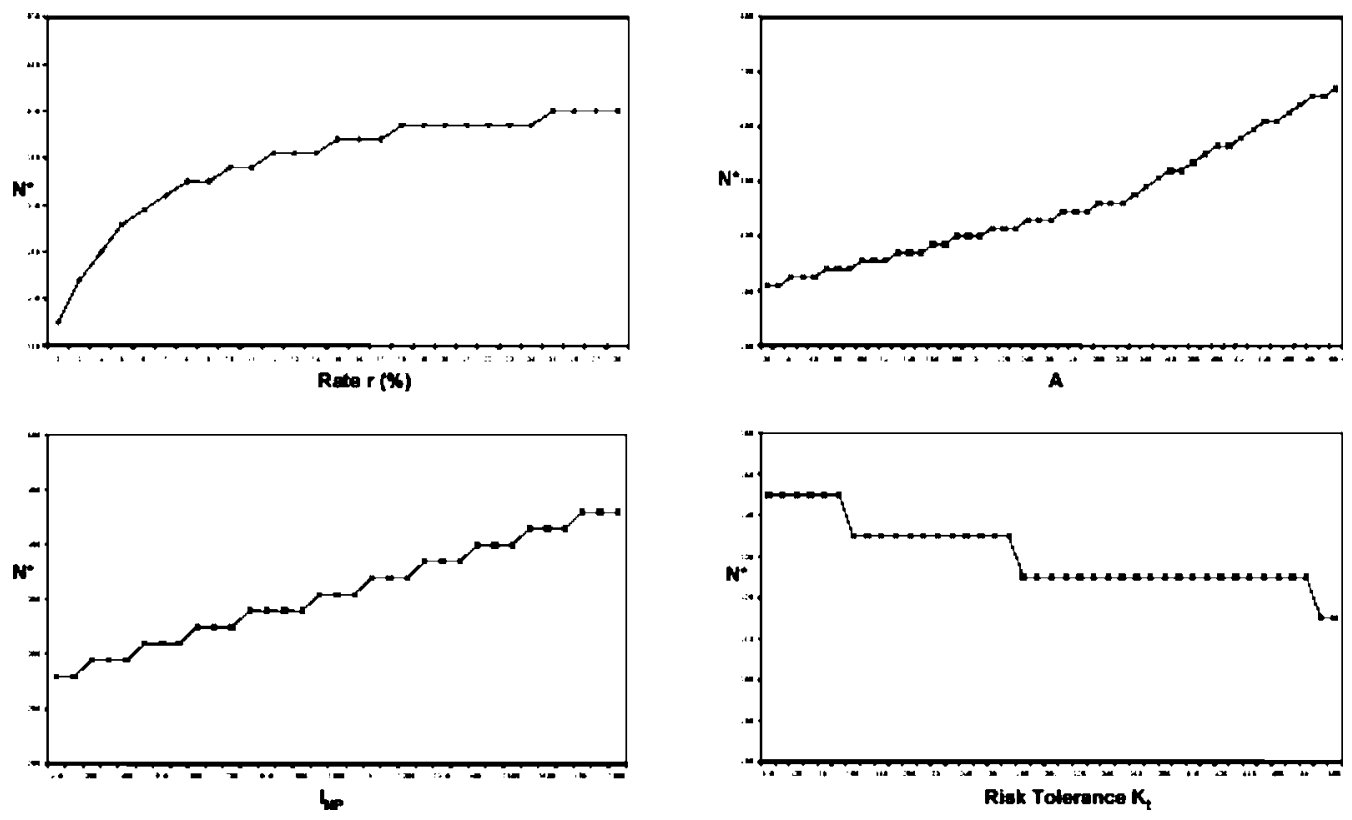

Figure 8. Sensitivity analysis for $N_{0}^{*}$.

$N_{0}^{*}$ increases with $A, I_{N}$ and also with $r$. The latter relation has its economic foundation in the idea that very long term projects are usually more sensitive to an increase in the discount factor. Finally, the relationship between $N_{0}^{*}$ and the effective risk tolerance factor is analogous to the one between the factor and $P_{0}^{*}$.

\section{Conclusions}

In this paper we have taken into account the economic as well as the environmental aspect of irreversibility. While the former feature has been largely investigated in the quasi-option value literature, the latter has been often ignored.

However, particularly in European applications of land allocation problems, the importance of initial environmental expenditures should be considered, combining it with the potential flexibility of this option. Moreover, the uncertainty related to the environmental response to recovery and conservation interventions, should be taken into account in addition to the uncertainty related to market prices. Therefore, the valuation procedure has been developed to include the environmental influence on the conservation projects. What we find is that it is possible to calculate, in a similar framework to the Arrow-Fisher Quasi-Option Value (QOV) of preservation, an option value that can be related either to the $D V$ or to the $N P$ decisions. Hence, the model capturing the flexibility, enables us to take into account different sources of irreversibility and uncertainty, and it proves to be a useful tool in land valuation, land allocation strategies and environmental expenditure management. In addition, the output of the model can be easily understood 
by non-technical users and it can be helpful in promoting environmental and economical rationale choices.

\section{Acknowledgments}

The authors thank two referees for their comments and suggestions on the original version of the paper.

\section{Notes}

1. This is clearly an oversimplification of the complexity of possible environmental quality scenarios, however the assumptions are useful for explanatory purposes and may be easily modified to provide a more realistic representation of environmental quality.

2. This particular result derives from the nature of the price process, that is assumed to be a discrete martingale. Therefore, $E\left[P_{t+1}-P_{t}\right]=E\left[\Delta P_{t+1, t}\right]=0$ such that the following expression is required $q(u-1)+(1-q)(d-1)=E\left[\Delta P_{t+1, t}\right]=0$.

3. The decision maker's preferences for risky cash flows depend only on the marginal distribution for each period, not on the joint distribution, see appendix in (Smith and Nau, 1995).

4. If the decision maker is indifferent to a gamble or to its certainty equivalent, then she will be indifferent to the same gamble plus a quantity $\Delta$ or the certainty equivalent of the gamble plus the same quantity $\Delta$, see appendix in (Smith and Nau, 1995).

\section{References}

Albers, H. (1996). "Modeling Ecological Constraints on tropical Forest Management: Spatial Interdependence, Irreversibility and Uncertainty." Journal of Environmental Economics 30, 73-94.

Arrow, K.J. and A.C. Fisher. (1974). "Environmental Preservation, Uncertainty, and Irreversibility." Quarterly Journal of Economics 88, 312-319.

Coggins, J.S. and C.A. Ramezani. (1998). “An Arbitrage-Free Approach to Quasi-Option Value.” Journal of Environmental Economics and Management 35, 103-125.

Conrad, J. (2000). "Wilderness: Options to Preserve, Extract, or Develop." Resources and Energy Economics 22, 205-219.

Conrad, J. (1980). "Quasi-Option Value and the Expected Value of Information." Quarterly Journal of Economics 94, 813-820.

Cortazar, G., E. Schwartz, and M. Salinas. (1998). "Evaluating Environmental Investments: A Real Options Approach." Management Science 44(8), 1059-1070.

Dixit, A.K. and R.S. Pindyck. (1994). Investment Under Uncertainty. Princeton, NY: Princeton University Press.

Dixit, A.K. and R.S. Pindyck. (1995). "The Options Approach to Capital Investment." Harvard Business Review, May-June.

Eichberger, J. and I.R. Harper. (1997). Financial Economics, chapters 3-4. Oxford: Oxford University Press.

Gaston, K.J. et al. (1998). Conservation Science and Action, W.J. Sutherland (ed.), C.J. Bibby, Selecting Area for Conservation, chapter 9.

Hanemann, W.M. (1989). "Information and the Concept of Option Value." Journal of Environmental Economics and Management 16, 23-37. 
Krutilla, J.V. and A.C. Fisher.(1975). The Economics of Natural Environments: Studies in the Valuation of Commodity and Amenity Resources. Baltimore, MD: The Johns Hopkins University Press.

Kolstad, C.D. (1996). "Learning, Irreversibilities and Climate." In Proceedings of the 7th EAERE Conference, Lisbon, Portugal.

Merton, R.C. (1994). Continuous-Time Finance. Cambridge/Oxford: Blackwell.

Oksendal, B. and H. Yaozhong. (1998). "Optimal Time to Invest when the Price Processes are Geometric Brownian Motions." Finance and Stochastics 2, 295-310.

Pindyck, R.S. (2000). "Irreversibility and the Timing of Environmental Policy." Working Paper 5, FEEM.

Smith, J.E. and R.F. Nau. (1995). "Valuing Risky Projects: Option Pricing Theory and Decision Analysis." Management Science 41, 795-816.

Smith, J.E and K.F. McCardle. (1998). "Valuing Oil Properties: Integrating Option Pricing and Decision Analysis Approaches." Operations Research 46, 198-217.

Smith, J.E and K.F. McCardle. (1999). "Options in Real World: Lessons Learned in Evaluating Oil and Gas Investment." Operations Research 47, 1-15.

Trigeorgis, L. (1996). Real Options: Managerial Flexibility and Strategy in Resources Allocation. Cambridge, MA: MIT Press. 